\title{
Interferometer for force measurement via a shortcut to adiabatic arm guiding
}

\author{
A. Rodriguez-Prieto, ${ }^{1}$ S. Martínez-Garaot $\odot,{ }^{2}$ I. Lizuain $\odot,{ }^{3}$ and J. G. Muga $\odot^{2}$ \\ ${ }^{1}$ Departament of Applied Mathematics, University of the Basque Country UPV/EHU, Bilbao 48013, Spain \\ ${ }^{2}$ Departament of Physical Chemistry, University of the Basque Country UPV/EHU, Apartado 644, Bilbao 48080, Spain \\ ${ }^{3}$ Departament of Applied Mathematics, University of the Basque Country UPV/EHU, Donostia-San Sebastián 20018, Spain
}

(Received 10 March 2020; accepted 20 May 2020; published 12 June 2020; corrected 17 September 2020)

\begin{abstract}
We propose a compact atom interferometer to measure homogeneous constant forces guiding the arms via shortcuts to adiabatic paths. For a given sensitivity, which only depends on the space-time area of the guiding paths, the cycle time can be made fast without losing visibility. The atom is driven by spin-dependent trapping potentials moving in opposite directions, complemented by linear and time-dependent potentials that compensate the trap acceleration. Thus, the arm states are adiabatic in the moving frames and nonadiabatic in the laboratory frame. The trapping potentials may be anharmonic, e.g., optical lattices, and the interferometric phase does not depend on the initial motional state or on the pivot point for swaying the linear potentials.
\end{abstract}

DOI: 10.1103/PhysRevResearch.2.023328

\section{INTRODUCTION}

Atom interferometry [1,2] provides a route to quantumenhanced precise sensors. The key idea is to split and later recombine the atom wave function, to detect the differential phase accumulated during the separation, which is sensitive, in particular, to small potential differences between the arms.

Here, we work out a scheme to measure constant forces using STA-mediated guided interferometry [3-6]. STA stands for "shortcuts to adiabaticity," a set of techniques to achieve the results of adiabatic dynamics in shorter times [7,8], and "guided" means that the atom is driven in moving traps as in buckets or conveyor belts [5], so it is never in free flight. Guiding, e.g., via moving optical lattices, keeps the atom wave function localized with nanoscale spatial resolution, allowing for precise measurements at the ultrashort spatial scale $[9,10]$, whereas the speedup with respect to slow adiabatic processes can avoid perturbations and decoherence keeping the visibility and differential phase of adiabatic methods. Moreover, our STA-mediated interferometer scheme fulfills the ideal goal of giving a motional-state independent differential phase with short process times whereas keeping simultaneously a high sensitivity.

We assume throughout one-particle wave functions either because the interferometer works, indeed, with single particles or because interactions are negligible. Similar to Ref. [3] the arms in the current scheme are separated by means of "spin," here, an alias for "internal state"-dependent forces. Operationally, the current scheme differs from the one in Ref. [3]. There, a fixed harmonic trap was combined with

Published by the American Physical Society under the terms of the Creative Commons Attribution 4.0 International license. Further distribution of this work must maintain attribution to the author(s) and the published article's title, journal citation, and DOI. two homogeneous time- and spin-dependent forces to separate first and then recombine the wave-function branches of an ion. Here, we use, instead, two moving spin-dependent traps, not necessarily harmonic, complemented by homogeneous spin- and time-dependent forces to compensate for inertial terms due to the motions of the traps [11]. This compensation is one of the ways to implement STA-driven fast transport [8] and can be equivalently found by invariant-based inverse engineering [11], by the "fast-forward approach" [12], or as a local unitary transformation of a nonlocal counterdiabatic approach $[13,14]$.

An important difference between this paper and Ref. [3] is that the phase differential is now independent of the pivot equipotential point $x_{0}$ to apply the compensating spindependent potentials, see an example of two different pivot positions in the outer columns of Fig. 1. When the force to be measured acts permanently, before and during the experiment, the natural choice in which $x_{0}$ is at the initial equilibrium point of the trap, which depends on the unknown force we want to measure, canceled the interferometric phase in Ref. [3]. A rotation of the effectively one-dimensional trap to let the force act only from the initial time $t=0$ is a formal but hardly practical solution. The scheme proposed here is free from such difficulties and is more broadly applicable.

Using arbitrary trapping potentials rather than harmonic ones opens the way to apply the proposed scheme to ultracold neutral atoms where the anharmonicities are usually stronger than for trapped ions. Different realizations are possible, e.g., in atom chips [2], but we will discuss optical lattices as a specific example. Interferometers with two oppositely moving optical lattices to accelerate the arm wave functions for a single internal state have been demonstrated [15] and studied theoretically [9]. Closer to our goal, Mandel et al. [16] demonstrated transport of the spin-dependent wave functions in optical lattices moving in opposite directions with a scheme proposed in Ref. [17], and Steffen et al. [10] built a singleatom interferometer based on a similar setting. To implement 


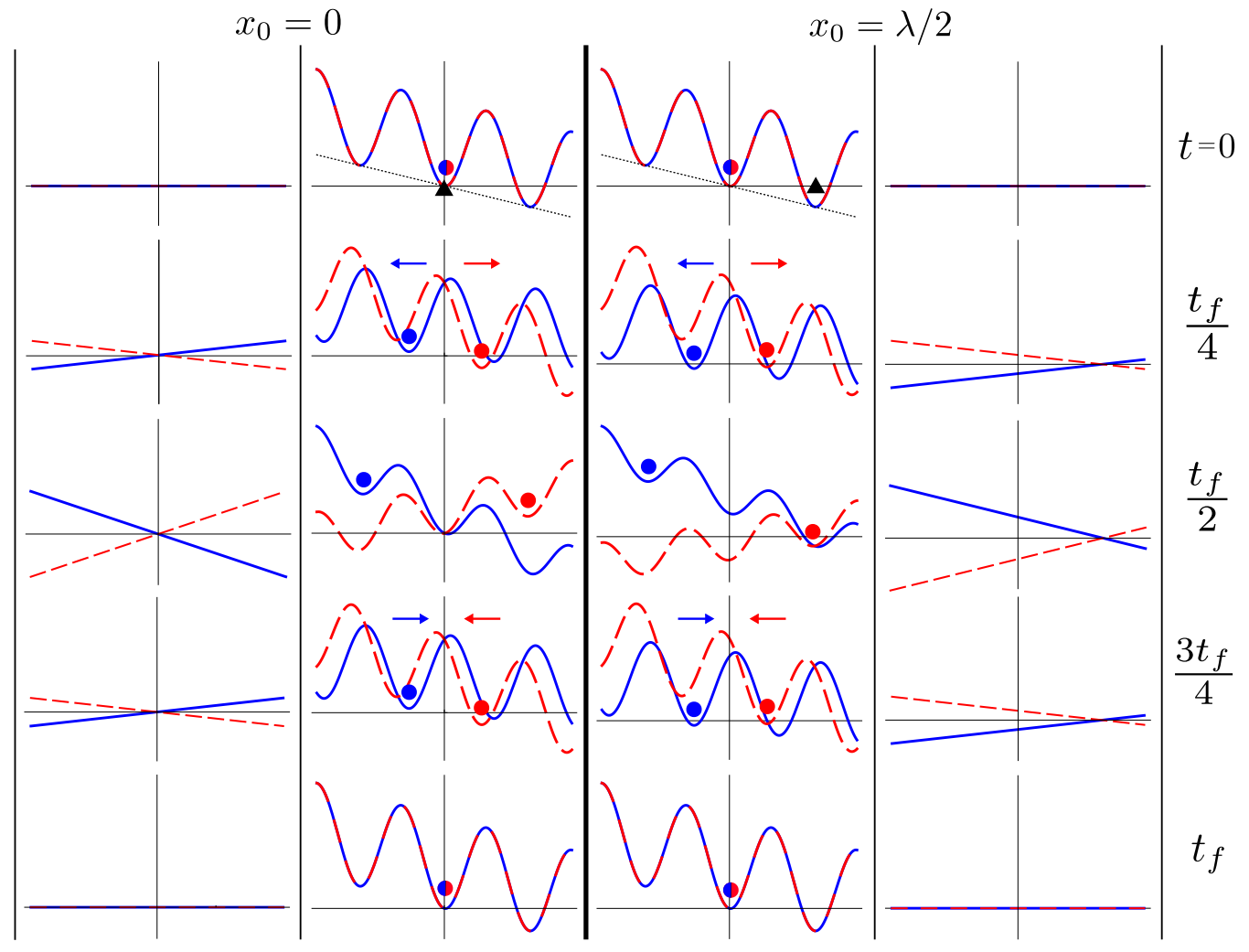

FIG. 1. Four schematic snapshots of the potentials for moving optical lattices and two choices of pivot $x_{0}$. We use arbitrary units, the specific relative values are not intended to be realistic but are rather chosen to better visualize the process. The corresponding $\alpha(t)$ and $f(t)$ are shown in Fig. 3. $U(x \mp \alpha)=U_{0} \sin ^{2}(2 \pi x / \lambda \mp \alpha)$. The two left columns are for $x_{0}=0$, and the two right columns are for $x_{0}=\lambda / 2$, a lattice period to the right. Outer columns: compensating potentials for spin-up $-\left(x-x_{0}\right) f(t)$ (red dashed line) and for spin-down $\left(x-x_{0}\right) f(t)$ (blue solid line). In the central columns, the triangles are a reminder of the pivot position, and the points indicate the moving $\pm \alpha(t)$. The arrows give the sense of motion of the lattice. $-c x$ (dotted black line) is represented only at $t=0$, but this spin-independent potential acts throughout the process; the wavy lines are the total potentials in Eq. (3), $U(x-\alpha)-\left(x-x_{0}\right) f(t)-c x$ for spin up (red dashed line) and $U(x+\alpha)+\left(x-x_{0}\right) f(t)-c x$ for spin down (solid blue).

our scheme, we envision, for each spin, double superlattices composed by an ultradeep optical lattice as a "conveyor-belt" trapping potential [18] with negligible tunneling, see Ref. [19] and references therein, whereas the compensating force may be achieved by a second lattice with much larger periodicity than the trapping lattice to make it effectively homogeneous for each arm wave function. Factors of 10 between the periodicities of two lattices are routinely found playing with the angle between the crossing beams [20], and even higher factors are technically possible [21].

First, we present the main idea of the interferometer and basic relations in Sec. II and, then, the recipe to move the arm traps and set the time dependence of the compensating forces in Sec. III. The theory relies on a transformation to the "moving-frame" interaction pictures for each arm. An alternative formulation is presented in Sec. IV in terms of "invariants" which connects the current approach to "invariant-based inverse engineering" $[3,6,11]$. The interferometric phase can then be simply interpreted as the difference between the Lewis-Riesenfeld phases for the arms. This connection enables us to use invariant-based concepts and results, for example, to apply techniques to enhance robustness with respect to different noises [19,22,23]. The paper ends with a discussion on possible applications and open questions.

\section{BASIC CONCEPT OF THE INTERFEROMETER}

Consider an atom with two internal states, "spin-up" $|\uparrow\rangle$, and "spin-down" $|\downarrow\rangle$, and effective motion in one dimension. A general state at time $t$ is $a_{\uparrow}|\uparrow\rangle \psi^{\uparrow}(x, t)+a_{\downarrow}|\downarrow\rangle \psi^{\downarrow}(x, t)$, where $\psi^{\uparrow}(x, t)=\left\langle x \mid \psi^{\uparrow}(t)\right\rangle$ and $\psi^{\downarrow}(x, t)=\left\langle x \mid \psi^{\downarrow}(t)\right\rangle$ are the motional states for the two internal levels in coordinate representation. We assume a prepared state $|\uparrow\rangle\left|\Phi_{p}\right\rangle$ from which a $\pi / 2$ pulse [24] produces two equally weighted components with $a_{\uparrow}=a_{\downarrow}=1 / 2^{1 / 2}$. We set time $t=0$ at the end of the $\pi / 2$ pulse and, assuming a Lamb-Dicke regime and a fast pulse compared to motional periods, $\Phi(x, 0) \equiv \Phi_{p}(x)=$ $\psi^{\uparrow}(x, 0)=\psi^{\downarrow}(x, 0)$. The two branches evolve separately in coordinate space due to spin-dependent forces. At some final time $t_{f}$, the complex overlap can be written in polar form as

$$
\left\langle\psi^{\downarrow}\left(t_{f}\right) \mid \psi^{\uparrow}\left(t_{f}\right)\right\rangle=e^{i \Delta \varphi\left(t_{f}\right)}\left|\left\langle\psi^{\downarrow}\left(t_{f}\right) \mid \psi^{\uparrow}\left(t_{f}\right)\right\rangle\right| .
$$

A second $\pi / 2$ pulse gives the populations [3],

$$
P^{\uparrow \downarrow}\left(t_{f}\right)=\frac{1}{2} \pm \frac{1}{2} \operatorname{Re}\left[\left\langle\psi^{\downarrow}\left(t_{f}\right) \mid \psi^{\uparrow}\left(t_{f}\right)\right\rangle\right],
$$

where we have neglected the $\pi / 2$-pulse duration. The STA driving will achieve maximal visibility, i.e., $\left|\left\langle\psi^{\downarrow}\left(t_{f}\right) \mid \psi^{\uparrow}\left(t_{f}\right)\right\rangle\right|=1$, note that $\left|\left\langle\psi^{\uparrow \downarrow}\left(t_{f}\right) \mid \Phi(0)\right\rangle\right|=$ 1 is not required. Then, the populations read 
$P^{\uparrow \downarrow}\left(t_{f}\right)=\frac{1}{2} \pm \frac{1}{2} \cos \left[\Delta \varphi\left(t_{f}\right)\right]$. If the differential phase is proportional to a constant force $c, \Delta \varphi\left(t_{f}\right)=\mathcal{S} c$, and the sensitivity $\mathcal{S}$ is known, $c$ can be measured from the populations. When $c$, or its deviation from some approximately known value, are expected to be small in the scale of $\pi / \mathcal{S}, c$ is found from the populations using the relevant branch of the arccosine. More generally, $c$ may be found unambiguously from the periodicity $2 \pi / c$ of the populations $P^{\uparrow \downarrow}\left(t_{f}\right)$ as a function of $\mathcal{S}$ [3]. Measuring the populations requires repetitions in time if the interferometer works with a single particle, or alternatively, noninteracting ensembles.

The method to guide the arm wave functions described below fulfills the hypotheses made so far, namely, the modulus of the overlap (1) is one, and the differential phase is proportional to $c$. Moreover, it will be possible to control the sensitivity $\mathcal{S}$, and the time $t_{f}$ of the process independently of $|\Phi(0)\rangle$.

\section{HOW TO MOVE THE GUIDING TRAPS}

For each spin state, we assume a different evolution driven by the Hamiltonians, (here, and in the following, the superscript $\uparrow \downarrow$ in any equation implies that the sign on top in $\mp$ or \pm is for $\uparrow$, whereas the sign on the bottom is for $\downarrow$ )

$$
H^{\uparrow \downarrow}=\frac{p^{2}}{2 m}-c x \mp\left[x-x_{0}(t)\right] f(t)+U[x \mp \alpha(t)] .
$$

The trap potentials $U[x \mp \alpha(t)]$ move along opposite trajectories $\alpha^{\uparrow \downarrow}(t)= \pm \alpha(t)$. We consider trap trajectories that satisfy the boundary conditions

$$
\alpha\left(t_{b}\right)=\dot{\alpha}\left(t_{b}\right)=0,
$$

at the boundary times $t_{b}=0, t_{f}$. The dots stand for time derivatives. Each trap starts and ends at rest returning to the starting point, equal for both traps.

The trap potentials are complemented by spin-dependent linear potentials $\mp\left[x-x_{0}(t)\right] f(t)$ that cross at the pivot point $x_{0}(t)$ : In a typical experiment, $x_{0}$ will be constant, however, we will keep, by now, formally a more general $x_{0}(t)$. The force $f(t)$ will be chosen to compensate inertial terms in the moving frame as discussed below in detail. Finally, $c$ is the spin-independent homogeneous-in-space and constant-intime force that we want to measure, $m$ is the mass of the atom, and $p^{2} / 2 m$ is the kinetic energy. Examples of the potential terms in Eq. (3) are depicted schematically in Fig. 1 for $U$ as an optical lattice potential and for two different pivots.

Let us now reorganize the Hamiltonians (3) as follows:

$$
H^{\uparrow \downarrow}=\frac{p^{2}}{2 m} \mp f(t) x+\widetilde{U}(x \mp \alpha)+\Lambda^{\uparrow \downarrow}(t),
$$

where we have separated purely time-dependent terms in

$$
\Lambda^{\uparrow \downarrow}(t)= \pm f(t) x_{0}(t) \mp c \alpha(t),
$$

and defined effective trap potentials $\widetilde{U}$ that include the effect of the force $c$,

$$
\widetilde{U}[x \mp \alpha(t)]=U[x \mp \alpha(t)]-[x \mp \alpha(t)] c .
$$

To solve the dynamics, it is useful to perform unitary transformations into "moving-frame interaction pictures." Specif- ically we define the interaction picture wave-vectors $\left|\psi_{I}^{\uparrow \downarrow}\right\rangle$ in terms of Schrödinger (laboratory frame) wave-vectors $\left|\psi^{\uparrow \downarrow}\right\rangle$ as

$$
\left|\psi_{I}^{\uparrow \downarrow}\right\rangle=\mathcal{U}^{\uparrow \downarrow}\left|\psi^{\uparrow \downarrow}\right\rangle, \quad\left|\psi^{\uparrow \downarrow}\right\rangle=\left(\mathcal{U}^{\uparrow \downarrow}\right)^{\dagger}\left|\psi_{I}^{\uparrow \downarrow}\right\rangle,
$$

where the unitary operator $\mathcal{U}^{\uparrow \downarrow}$ is constructed by multiplying position and momentum shift operators,

$$
\mathcal{U}^{\uparrow \downarrow}=e^{ \pm i \alpha p / \hbar} e^{\mp i m \dot{\alpha} x / \hbar} .
$$

Other orderings and, therefore, interaction pictures are possible but the measurable quantities and the differential phase are not affected by the different orderings as long as the intermediate calculations are performed consistently. Using Eq. (9) for each arm, the effective moving-frame Hamiltonians become

$$
\begin{aligned}
H_{I}^{\uparrow \downarrow}= & \mathcal{U}^{\uparrow \downarrow} H^{\uparrow \downarrow}\left(\mathcal{U}^{\uparrow \downarrow}\right)^{\dagger}+i \hbar \dot{\mathcal{U}}^{\uparrow \downarrow}\left(\mathcal{U}^{\uparrow \downarrow}\right)^{\dagger} \\
= & \frac{p^{2}}{2 m}+\frac{1}{2} m \dot{\alpha}^{2} \mp(x \pm \alpha) f(t)+\widetilde{U}(x) \\
& \pm f(t) x_{0}(t) \mp c \alpha \pm(x \pm \alpha) m \ddot{\alpha} .
\end{aligned}
$$

If the applied $f(t)$ satisfies

$$
\ddot{\alpha}(t)=\frac{f(t)}{m},
$$

which can be interpreted as a Newton equation for the trajectory $\alpha(t)$ subjected to the force $f(t)$, this auxiliary force compensates for inertial effects due to the motion of the $\widetilde{U}[x \mp$ $\alpha(t)$ ] potentials in the laboratory frame, note the cancellation of the third and last terms in Eq. (10). The consequence is that a stationary state in the moving frame will remain so. Equation (11) is used inversely, i.e., $f(t)$ is found from a designed $\alpha(t)$ and, hereinafter, $f(t)$ is always assumed to satisfy Eq. (11) except in point (vii) of the final discussion. To make $f\left(t_{b}\right)$ zero at the boundary times, we will impose, in addition to the boundary conditions (4), that $\ddot{\alpha}\left(t_{b}\right)=0$. Applying Eq. (11) in Eq. (10), the moving-frame Hamiltonians take a simple form with a common time- and spin-independent term $H_{I, 0}$ and terms $F^{\uparrow \downarrow}(t)$ that depend on time but not on $x$ or $p$,

$$
\begin{aligned}
H_{I}^{\uparrow \downarrow} & =H_{I, 0}+F^{\uparrow \downarrow}(t), \quad H_{I, 0}=\frac{p^{2}}{2 m}+\widetilde{U}(x), \\
F^{\uparrow \downarrow}(t) & =\frac{1}{2} m \dot{\alpha}^{2} \pm f(t) x_{0}(t) \mp c \alpha(t) .
\end{aligned}
$$

The resulting structure facilitates the formal solution of the dynamics as the time-dependent part only accumulates a phase, whereas the time-independent part gives a simple evolution operator,

$$
\begin{aligned}
& \left|\psi_{I}^{\uparrow \downarrow}(t)\right\rangle=\exp \left(-i \int_{0}^{t} F^{\uparrow \downarrow}\left(t^{\prime}\right) d t^{\prime} / \hbar\right)\left|\psi_{I, 0}^{\uparrow \downarrow}(t)\right\rangle, \\
& \left|\psi_{I, 0}^{\uparrow \downarrow}(t)\right\rangle=e^{-i H_{I, 0} t / \hbar}\left|\psi_{I, 0}^{\uparrow \downarrow}(0)\right\rangle .
\end{aligned}
$$

As $\left|\psi_{I, 0}^{\uparrow \downarrow}(0)\right\rangle=|\Phi(0)\rangle$ and $H_{I, 0}$ are spin independent, $\left|\psi_{I, 0}^{\uparrow \downarrow}(t)\right\rangle=|\Phi(t)\rangle=e^{-i H_{I, 0} t / \hbar}|\Phi(0)\rangle \quad$ is also a spinindependent vector.

Noting that $e^{\mp i \alpha p / \hbar}$ shifts the position representation as $\left\langle x\left|e^{\mp i \alpha p / \hbar}\right| \Phi\right\rangle=\Phi(x \mp \alpha)$, the branch wave functions in the 
laboratory frame are found by Eq. (8),

$$
\psi^{\uparrow \downarrow}(x, t)=e^{ \pm i m \dot{\alpha} x / \hbar} \exp \left(-i \int_{0}^{t} F^{\uparrow \downarrow}\left(t^{\prime}\right) d t^{\prime} / \hbar\right) \Phi(x \mp \alpha, t) .
$$

In particular, at final time $t_{f}$,

$$
\begin{aligned}
\psi^{\uparrow \downarrow}\left(x, t_{f}\right)= & \exp \left(-i m \int_{0}^{t_{f}} \dot{\alpha}^{2} d t /(2 \hbar)\right) \\
& \times \exp \left( \pm i c \int_{0}^{t_{f}} \alpha(t) d t / \hbar\right) \\
& \times \exp \left(\mp i \int_{0}^{t_{f}} x_{0}(t) f(t) d t / \hbar\right) \Phi\left(x, t_{f}\right) .
\end{aligned}
$$

For $x_{0}$ constant, but otherwise arbitrary, the overlap in Eq. (1) takes a very simple form since the phase terms $\mp x_{0} \int_{0}^{t_{f}} f(t) d t=0$ vanish because of Eq. (11) and the boundary condition $\dot{\alpha}\left(t_{b}\right)=0$,

$$
\left\langle\psi^{\downarrow}\left(t_{f}\right) \mid \psi^{\uparrow}\left(t_{f}\right)\right\rangle=\exp \left(2 i c \int_{0}^{t_{f}} \alpha(t) d t / \hbar\right),
$$

so that $c$ can be measured from the interferometric differential phase via the populations as explained before. The phase is, indeed, proportional to $c, \Delta \varphi\left(t_{f}\right)=c \mathcal{S}$ with controllable sensitivity,

$$
\mathcal{S}=\frac{2}{\hbar} \int_{0}^{t_{f}} \alpha(t) d t
$$

the space-time area $2 \int_{0}^{t_{f}} \alpha(t) d t$ in units of $\hbar$ swept between the two trap paths. Because the relative motion of the motional states with respect to $\pm \alpha(t)$ is identical in both arms, this is the same area between the state centroids for any initial motional state $|\Phi(0)\rangle$. Thus, the interferometric phase and sensitivity are independent of the initial motional state, a robust "geometrical" feature of the proposed interferometer.

$t_{f}$ can be chosen freely, in particular, it can be made short compared to relevant decoherence times, and $\alpha(t)$ can be manipulated to change the sensitivity. Examples on how to set $\alpha(t)$ may be found in Ref. [3], the basic idea is to expand it in some basis, e.g., sines or powers of $t$ with enough number of terms to satisfy the boundary conditions. More terms are added if further conditions are imposed, such as a desired value of $\mathcal{S}$.

\section{Example: Sensitivity for the cesium atom interferometer}

Steffen et al. [10] implemented a cesium atom interferometer which demonstrates some elements of the current scheme, specifically the atom wave function was split into separated paths controlled by spin-dependent optical-lattice potentials. A large displacement of $\pm \alpha(t)$ was technicallynot fundamentally-limited by the maximum voltage that can be applied to an electro-optical modulator $[10,16]$. This limitation was circumvented by accumulating elementary operation blocks which consist of lattice displacements with alternating directions interleaved by $\pi$ pulses $[10,16]$. A single lattice displacement by $\lambda / 4$ took $18 \mu$ s. Figure 2 represents a contour plot of the sensitivities (17) with a four-term polynomial $\alpha(t)$ satisfying the boundary conditions (4) and $\ddot{\alpha}\left(t_{b}\right)=0$

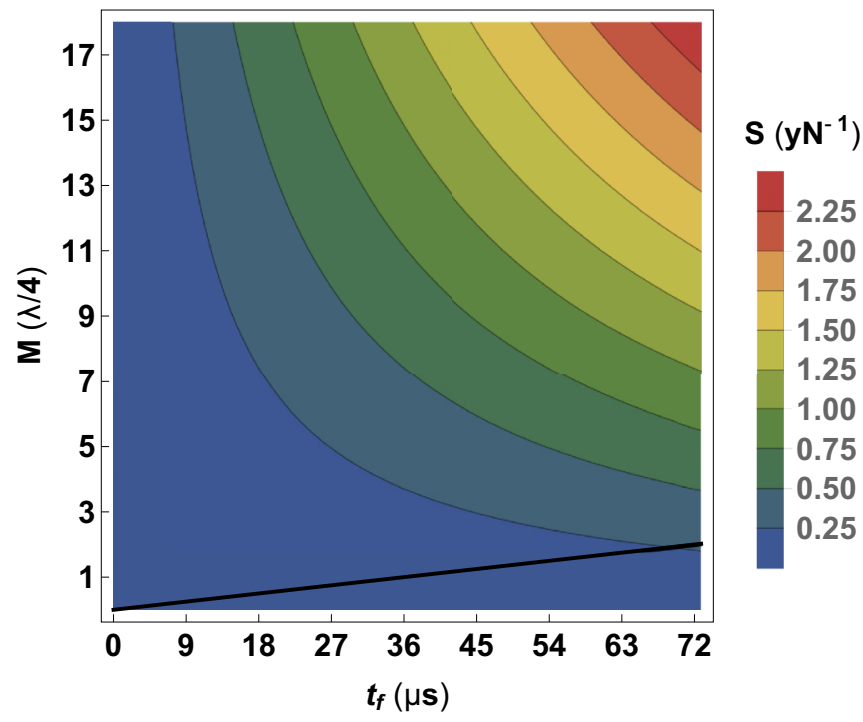

FIG. 2. Contour plot of the sensitivity $\mathcal{S}=\frac{32 M t_{f}}{35 \hbar}$ found for $\alpha(t)$ in Eq. (18), see also Fig. 3. The solid black line is a linear dependence of $M=\frac{\lambda}{72 \mu s} \frac{t_{f}}{2}$ that extrapolates the one applied in Ref. [10] for an elementary displacement. $\lambda=866 \mathrm{~nm}$.

with its maximum value $M$ at $t_{f} / 2$, see Fig. 3,

$$
\begin{aligned}
\alpha(t) & =\sum_{j=3}^{6} b_{j}\left(\frac{t}{t_{f}}\right)^{j}, \\
b_{3} & =64 M, \quad b_{4}=-192 M, \\
b_{5} & =192 M, \quad b_{6}=-64 M .
\end{aligned}
$$

The resulting sensitivity $\mathcal{S}$ is remarkably simple, namely, $\mathcal{S}=$ $32 M t_{f} /(35 \hbar)$. Note that the scaling of $\mathcal{S}$ with $t_{f}$ can be chosen at will by fixing the dependence of $M$ on $t_{f}$, this amounts to follow a line $M\left(t_{f}\right)$ in Fig. 2, for example, as $M \propto t_{f}^{\mu}$ with $\mu=0,1,2, \ldots$ Assuming a dependence of the order of the elementary displacement in Ref. [10] gives $M=\frac{\lambda}{72 \mu \mathrm{s}} \frac{t_{f}}{2}$, see the straight line in Fig. 2, and an $\mathcal{S}$ that depends quadratically on $t_{f}$. With the current scheme, the trap can be subjected to strong accelerations without spoiling the visibility since they are compensated. Thus, for a given $t_{f}$, higher sensitivities can

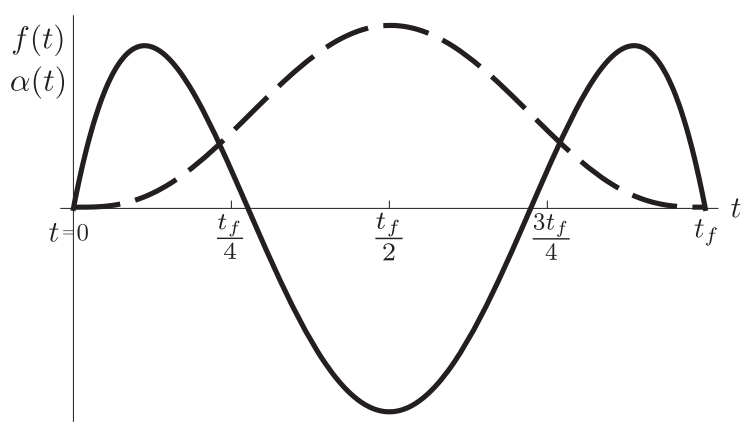

FIG. 3. Typical forms of $\alpha(t)$ (dashed line) and $f(t)$ (solid line) in arbitrary units: $\alpha(t)$ is found by imposing the boundary conditions (4) and $\ddot{\alpha}\left(t_{b}\right)=0$ at times $t_{b}=0, t_{f}$ to a polynomial ansatz [3], see Eq. (18). $f(t)$ is found from $\alpha(t)$ via Eq. (11), it vanishes at the boundary times and integrates to zero. 
be achieved for faster dependences $M\left(t_{f}\right)$. Formally, there is no limit to how large $\mathcal{S}\left(t_{f}\right)$ may be. The limit will be set, in practice, by the technical limitations imposed by the specific setting to implement $\alpha(t)$ and $f(t)$. For a given, desired sensitivity $\mathcal{S}_{0}, M\left(t_{f} ; \mathcal{S}_{0}\right)$ depends, along a given contour in Fig. 2, inversely on $t_{f}, M\left(t_{f} ; \mathcal{S}_{0}\right)=35 \mathcal{S}_{0} \hbar /\left(32 t_{f}\right)$. If $M$ is technically limited by some upper value, $t_{f}$ will be lower limited accordingly.

\section{INVARIANTS}

In this section, we will connect the results found so far with Lewis-Riesenfeld invariants of motion and the inverse engineering of trap trajectories based on them [7,11].

A key result is the moving-frame Hamiltonian structure found in Eq. (12). The Hamiltonian $H_{I, 0}$ does not depend on time, and, therefore, its expectation value $\left\langle\Phi(t)\left|H_{I, 0}\right| \Phi(t)\right\rangle$ is constant. In the laboratory frame, making use of Eq. (8), this translates into

$$
\frac{d}{d t}\left\langle\psi^{\uparrow \downarrow}(t)\left|\left(\mathcal{U}^{\uparrow \downarrow}\right)^{\dagger} H_{0, I} \mathcal{U}^{\uparrow \downarrow}\right| \psi^{\uparrow \downarrow}(t)\right\rangle=0,
$$

or, in other words,

$$
I^{\uparrow \downarrow} \equiv\left(\mathcal{U}^{\uparrow \downarrow}\right)^{\dagger} H_{0, I} \mathcal{U}^{\uparrow \downarrow}
$$

are "dynamical" Lewis-Riesenfeld invariants of motion for, respectively, the branch Hamiltonians $H^{\uparrow \downarrow}$ in Eq. (3), supplemented by Eq. (11) to specify $f(t)$. They satisfy the invariance equations,

$$
\frac{d I^{\uparrow \downarrow}}{d t}=\frac{\partial I^{\uparrow \downarrow}}{\partial t}+\frac{1}{i \hbar}\left[I^{\uparrow \downarrow}, H^{\uparrow \downarrow}\right] .
$$

These invariants may be calculated explicitly with the aid of Eq. (9),

$$
I^{\uparrow \downarrow}=\frac{1}{2 m}(p \mp m \dot{\alpha})^{2}+\widetilde{U}(x \mp \alpha),
$$

and their (constant-in-time) eigenvalues $\lambda_{n}$ are nothing but the eigenvalues of $H_{I, 0}$,

$$
\left[\frac{-\hbar^{2}}{2 m} \frac{\partial^{2}}{\partial x^{2}}+\tilde{U}(x)\right] \phi_{n}(x)=\lambda_{n} \phi_{n}(x),
$$

where the $\phi_{n}(x)$ 's are the eigenfuctions of $H_{I, 0}$. They form a natural basis to expand $|\Phi(t)\rangle$ as

$$
\begin{aligned}
|\Phi(t)\rangle & =\sum_{n} e^{-i \lambda_{n} t / \hbar}\left|\phi_{n}\right\rangle c_{n}, \\
c_{n} & =\left\langle\phi_{n} \mid \Phi(0)\right\rangle,
\end{aligned}
$$

in terms of constant coefficients $c_{n}$. The vectors,

$$
\left|\psi_{n}^{\uparrow \downarrow}\right\rangle \equiv\left(\mathcal{U}^{\uparrow \downarrow}\right)^{\dagger}\left|\phi_{n}\right\rangle
$$

are eigenvectors of $I^{\uparrow \downarrow}$ with eigenvalue $\lambda_{n}$ since

$$
\begin{aligned}
I^{\uparrow \downarrow}\left|\psi_{n}^{\uparrow \downarrow}\right\rangle & =\left(\mathcal{U}^{\uparrow \downarrow}\right)^{\dagger} H_{I, 0} \mathcal{U}^{\uparrow \downarrow}\left(\mathcal{U}^{\uparrow \downarrow}\right)^{\dagger}\left|\phi_{n}\right\rangle \\
& =\lambda_{n}\left(\mathcal{U}^{\uparrow \downarrow}\right)^{\dagger}\left|\phi_{n}\right\rangle=\lambda_{n}\left|\psi_{n}^{\uparrow \downarrow}\right\rangle .
\end{aligned}
$$

Using the explicit form of $\mathcal{U}^{\uparrow \downarrow}$ in Eq. (9) their coordinate representation is

$$
\psi_{n}^{\uparrow \downarrow}(x, t)=e^{ \pm i m \dot{\alpha} x / \hbar} \phi_{n}(x \mp \alpha) .
$$

The "dynamical modes" are defined as orthogonal solutions of the time-dependent Schrödinger equations driven by $H^{\uparrow \downarrow}$ proportional to these eigenfunctions $e^{i \theta_{n}^{\uparrow \downarrow}(t)} \psi_{n}(x, t)$ where the Lewis-Riesenfeld phases $\theta_{n}^{\uparrow \downarrow}(t)$ are found from

$$
\frac{d \theta_{n}^{\uparrow \downarrow}(t)}{d t}=\frac{1}{\hbar}\left\langle\psi_{n}^{\uparrow \downarrow}(t)\left|i \hbar \frac{\partial}{\partial t}-H^{\uparrow \downarrow}\right| \psi_{n}^{\uparrow \downarrow}(t)\right\rangle,
$$

so that the Schrödinger equations are satisfied. Setting $\theta_{n}^{\uparrow \downarrow}(0)=0$, an explicit calculation gives, see the Appendix,

$$
\theta_{n}^{\uparrow \downarrow}(t)=\frac{-1}{\hbar} \int_{0}^{t}\left[\lambda_{n}+F^{\uparrow \downarrow}\left(t^{\prime}\right)\right] d t^{\prime} .
$$

Arbitrary wave-function solutions of the dynamics $\psi^{\uparrow \downarrow}(t)$ will combine these elementary solutions with constant coefficients. For the initial-state $|\Phi(0)\rangle, \psi^{\uparrow \downarrow}(x, t)=$ $\sum_{n} e^{i \theta_{n}^{\uparrow \downarrow}(t)} \psi_{n}(x, t) c_{n}$. Factoring out $n$-independent phase factors and summing over $n$ as in Eq. (24), the expression (14) and following results for $\psi^{\uparrow \downarrow}(x, t)$ in the main text are exactly recovered. The interferometric phase from this point of view is nothing but the difference between Lewis-Riesenfeld phases for the arms. Since the $n$-dependent part cancels out, the result is $n$ independent.

\section{DISCUSSION}

We have put forward a STA-mediated atomic interferometer scheme to measure homogeneous constant forces with spin- (internal-state-) dependent moving traps to guide the wave-function components along the two arms. The approach is robust in different ways:

(i) As the process can be performed fast, decoherence effects and perturbations can be mitigated or avoided without necessarily renouncing to some required sensitivity. For a caveat on the relation between process time and decoherence, see point (vi) below.

(ii) The moving trapping potentials may be anharmonic, so the method may be applied, in particular, to optical lattices as conveyor belts to drive the arms.

(iii) The motional initial wave function is arbitrary, there is no need to prepare a perfect ground state because the differential phase is not affected by the initial motional state.

(iv) The moving trap potentials are complemented by timedependent linear potentials that compensate inertial forces "rocking" on a pivot point $x_{0}$. The differential interferometric phase is simplified and made pivot independent when $\int_{0}^{t_{f}} x_{0}(t) f(t) d t=0$ in Eq. (15). The integral vanishes when $x_{0}$ is a constant because of the way $f(t)$ is constructed. This result is, in fact, robust with respect to typical forms of $x_{0}(t)$ : A noisy $x_{0}(t)$ with a zero-mean perturbation around its nominal constant value will give a vanishing integral as long as the correlation time is short compared to $t_{f}$. Other relevant dependence is an undesired linear drift, e.g., $x_{0}(t)=$ $a+b t$. For the linear term $b t$ integrating by parts and using the boundary conditions (4) gives a zero integral too. It may also be of interest in practice to set a spin-dependent $x_{0}(t)$. For example, for $x_{0}^{\uparrow \downarrow}(t)= \pm \alpha(t)$, the resulting integrals $\pm \int_{0}^{t_{f}}[ \pm \alpha(t)] f(t) d t$ would not vanish, but they would give the same phase for both arms, which makes the differential phase again pivot independent. 
(v) There is ample freedom to choose the trap paths $\pm \alpha(t)$ which are only subjected, apart from technical limitations, to satisfy some boundary conditions at initial and final times. This flexibility may be used to change the sensitivity $\mathcal{S}$. It also allows to achieve fast scalings of $\mathcal{S}$ with total time $t_{f}$, in principle, with an arbitrary power of $t_{f}$, in contrast to linear scaling with $t_{f}$ of Ramsey-Bordé interferometers or with $t_{f}^{2}$ in a Mach-Zender configuration [25].

(vi) Following techniques developed to enhance the robustness of STA approaches [8,22], the freedom in choosing $\alpha(t)$ may be used to make the differential phase robust against specific setting-dependent perturbations, e.g., some particular type of noise relevant for the experimental arrangement. A recent study [19] analyzes the motional energy excitation of atoms due to noises affecting different moving optical lattice parameters: periodicity, depth, or position. The excitations may be analyzed in terms of static or dynamical contributions whose relative importance depends on the parameter affected by noise.

Static contributions are defined as those which are independent of the trap trajectory, they just increase with transport time $t_{f}$ so the strategy to mitigate them is to shorten process times. They are dominant, in particular, for position noise.

Dynamical contributions depend on the trap trajectory so they could be mitigated by a good choice of $\alpha(t)$. For "accordion noise" of the lattice periodicity, they dominate and give minimal excitation at a certain transport time. For noise in the trap depth, there is also a time $t_{f}$ with minimal excitation with dynamical terms dominating at shorter transport times and static terms at larger times.

The existence of minima-for some but not all noise types-underlines that the naive expectation that shorter and shorter times $t_{f}$ are always beneficial is not necessarily correct. The beneficial effect of shortening the time depends on the noise type and on the time domain. It also points out that there are no universal recipes, each noise or perturbation requires a dedicated study. Adapting the analysis in Ref. [19], which did not include compensating forces, to the current configuration, is left for a separate work.

(vii) The arm wave functions overlap, and the differential phases found in Eq. (16) are exact, i.e., no adiabatic approximation has been performed, and there is no need to calculate nonadiabatic corrections. In this regard, it is interesting to sketch how this result is found in the adiabatic slow motion limit when the compensating force $f(t)$ is not applied. The calculation would start in Eq. (10) for $H_{I}^{\uparrow \downarrow}$. Taking now $f(t)=0$, these moving-frame Hamiltonians cannot be separated into purely $t$-independent and purely $t$-independent terms because of the inertial terms $\pm x m \ddot{\alpha}$. In the slow-motion limit, however, these terms will be negligible compared to $\widetilde{U}$ so that the structure in that limit is again that of a time-independent Hamiltonian and purely time-dependent terms. The corresponding dynamics then lead to Eq. (16) but only as an approximation. In contrast, when the compensation forces $f(t)=\mp m \ddot{\alpha}$ are applied, the dynamics is generally nonadiabatic in the laboratory frame but adiabatic by construction in the moving frames a key property that allows us to set simultaneously short process times and large sensitivities.

We hope that the unique features of the proposed scheme, among them independence of the initial state, arbitrary trap potential, and freedom to choose sensitivity and cycle time will motivate further work. The elements necessary to implement the current scheme have been separately demonstrated. We have paid some attention to the use of oppositely moving spin-dependent optical lattices $[10,16]$. Alternative realizations may be based on the unitary equivalence between: (a) the "local", position-dependent compensating Hamiltonian terms $\mp m \ddot{\alpha}(t) x$; and (b) counterdiabatic momentum-dependent terms $\pm p \dot{\alpha}[8,13]$.Whereas implementing the former in the laboratory is quite generally easier than the latter, the spin-dependent counterdiabatic terms may be realized in systems with either actual or synthetic spin-orbit coupling [26-29].

\section{ACKNOWLEDGMENTS}

We thank D. Leibfried, J. Bollinger, and D. GuéryOdelin for many useful discussions. This work was supported by the Basque Country Government (Grant No. IT986-16) and by Grant No. PGC2018-101355-B-I00 (MCIU/AEI/FEDER,UE).

\section{APPENDIX: CALCULATION OF EQ. (29)}

To calculate the Lewis-Riesenfeld phases in Eq. (29), we start from calculating the matrix elements in Eq. (28). It proves convenient to write first, using Eqs. (10) and (12),

$$
H^{\uparrow \downarrow}=I^{\uparrow \downarrow}+F^{\uparrow \downarrow}-i \hbar\left(\mathcal{U}^{\uparrow \downarrow}\right)^{\dagger} \dot{\mathcal{U}}^{\uparrow \downarrow} .
$$

Using, now, Eq. (25) we find that

$-\left\langle\psi_{n}^{\uparrow \downarrow}\left|H^{\uparrow \downarrow}\right| \psi_{n}^{\uparrow \downarrow}\right\rangle / \hbar=\frac{-1}{\hbar}\left(\lambda_{n}+F^{\uparrow \downarrow}\right)+i\left\langle\phi_{n}\left|\dot{\mathcal{U}}^{\uparrow \downarrow}\left(\mathcal{U}^{\uparrow \downarrow}\right)^{\dagger}\right| \phi_{n}\right\rangle$,

whereas, using again Eq. (25) and noting that $\dot{\mathcal{U}}^{\uparrow \downarrow}\left(\mathcal{U}^{\uparrow \downarrow}\right)^{\dagger}=$ $-\mathcal{U}^{\uparrow \downarrow}\left(\dot{\mathcal{U}}^{\uparrow \downarrow}\right)^{\dagger}$

$$
i\left\langle\psi_{n}^{\uparrow \downarrow} \mid \dot{\psi}_{n}^{\uparrow \downarrow}\right\rangle=-i\left\langle\phi_{n}\left|\dot{\mathcal{U}}^{\uparrow \downarrow}\left(\mathcal{U}^{\uparrow \downarrow}\right)^{\dagger}\right| \phi_{n}\right\rangle .
$$

The right-hand side may be calculated explicitly, but, in any case, it is canceled by the last term in Eq. (A2) when summing Eqs. (A2) and (A3) in Eq. (28). Integrating, we get finally Eq. (29).
[1] P. R. Berman, Atom Interferometry (Academic, San Diego, 1997).

[2] A. D. Cronin, J. Schmiedmayer, and D. E. Pritchard, Rev. Mod. Phys. 81, 1051 (2009).

[3] S. Martínez-Garaot, A. Rodriguez-Prieto, and J. G. Muga, Phys. Rev. A 98, 043622 (2018).
[4] M. Dupont-Nivet, C. I. Westbrook, and S. Schwartz, New J. Phys. 18, 113012 (2016).

[5] P. Navez, S. Pandey, H. Mas, K. Poulios, T. Fernholtz, and W. von Kiltzing, New J. Phys. 18, 075014 (2016).

[6] M. Palmero, S. Martínez-Garaot, D. Leibfried, D. J. Wineland, and J. G. Muga, Phys. Rev. A 95, 022328 (2017). 
[7] E. Torrontegui, S. Ibáñez, S. Martínez-Garaot, M. Modugno, A. del Campo, D. Guéry-Odelin, A. Ruschhaupt, X. Chen, and J. G. Muga, Adv. At., Mol., Opt. Phys. 62, 117 (2013).

[8] D. Guéry-Odelin, A. Ruschhaupt, A. Kiely, E. Torrontegui, S. Martínez-Garaot, and J. G. Muga, Rev. Mod. Phys. 91, 045001 (2019).

[9] T. Kovachy, J. M. Hogan, D. M. S. Johnson, and M. A. Kasevich, Phys. Rev. A 82, 013638 (2010).

[10] A. Steffen, A. Alberti, A. W. Alt, N. Belmechri, S. Hild, M. Karski, A. Widera, and D. Meschede, Proc. Natl. Acad. Sci. USA 109, 9770 (2012).

[11] E. Torrontegui, S. Ibáñez, Xi Chen, A. Ruschhaupt, D. GuéryOdelin, and J. G. Muga, Phys. Rev. A 83, 013415 (2011).

[12] S. Masuda and K. Nakamura, Proc. R. Soc. A 466, 1135 (2010).

[13] S. Ibáñez, S. Martínez-Garaot, X. Chen, E. Torrontegui, and J. G. Muga, Phys. Rev. A 84, 023415 (2011); 86, 019901(E) (2012).

[14] S. Deffner, C. Jarzynski, and A. del Campo, Phys. Rev. X 4, 021013 (2014).

[15] H. Müller, S.-w. Chiow, S. Herrmann, and S. Chu, Phys. Rev. Lett. 102, 240403 (2009).

[16] O. Mandel, M. Greiner, A. Widera, T. Rom, T. W. Hänsch, and I. Bloch, Phys. Rev. Lett. 91, 010407 (2003).

[17] D. Jaksch, H.-J. Briegel, J. I. Cirac, C. W. Gardiner, and P. Zoller, Phys. Rev. Lett. 82, 1975 (1999).

[18] D. Schrader, S. Kuhr, W. Alt, M. Müller, V. Gomer, and D. Meschede, Appl. Phys. B: Lasers Opt. 73, 819 (2001).
[19] X.-J. Lu, A. Ruschhaupt, S. Martínez-Garaot, and J. G. Muga, Entropy 22, 262 (2020).

[20] R. A. Williams, J. D. Pillet, S. Al-Assam, B. Fletcher, M. Shotter, and C. J. Foot, Opt. Express 16, 16977 (2008).

[21] J. Tao, Y. Wang, Y. He, and S. Wu, Opt. Express 26, 14346 (2018).

[22] A. Ruschhaupt, Xi Chen, D. Alonso, and J. G. Muga, New J. Phys. 14, 093040 (2012).

[23] X. J. Lu, A. Ruschhaupt, and J. G. Muga, Phys. Rev. A 97, 053402 (2018).

[24] D. Leibfried, B. DeMarco, V. Meyer, D. Lucas, M. Barrett, J. Britton, W. M. Itano, B. Jelenković, C. Langer, T. Rosenband, and D. J. Wineland, Nature (London) 422, 412 (2003).

[25] G. D. McDonald, C. C. N. Kuhn, S. Bennetts, J. E. Debs, K. S. Hardman, J. D. Close, and N. P. Robins, Europhys. Lett. 105, 63001 (2014).

[26] Y. Ban, X. Chen, E. Ya Sherman, and J. G. Muga, Phys. Rev. Lett. 109, 206602 (2012); 109, 249901(E) (2012).

[27] T. Čadež, J. H. Jefferson, and Ramšak, New J. Phys. 15, 013029 (2013).

[28] T. Čadež, J. H. Jefferson, and Ramšak, Phys. Rev. Lett. 112, 150402 (2014).

[29] X. Chen, R.-L. Jiang, J. Li, Y. Ban, and E. Y. Sherman, Phys. Rev. A 97, 013631 (2018).

Correction: The previously published Fig. 1 contained an error in the outer column and has been replaced. 